\title{
Genome-wide DNA methylome analysis identifies methylation signatures associated with survival and drug resistance of ovarian cancers
}

\author{
David W. Chan ${ }^{1 *}$ D, Wai-Yip Lam², Fushun Chen', Mingo M. H. Yung ${ }^{1}$, Yau-Sang Chan ${ }^{1}$, Wai-Sun Chan', \\ Fangfang He${ }^{1}$, Stephanie S. Liu', Karen K. L. Chan' ${ }^{1}$, Benjamin Li ${ }^{2}$ and Hextan Y. S. Ngan ${ }^{1,3^{*}}$
}

\begin{abstract}
Background: In contrast to stable genetic events, epigenetic changes are highly plastic and play crucial roles in tumor evolution and development. Epithelial ovarian cancer (EOC) is a highly heterogeneous disease that is generally associated with poor prognosis and treatment failure. Profiling epigenome-wide DNA methylation status is therefore essential to better characterize the impact of epigenetic alterations on the heterogeneity of EOC.

Methods: An epigenome-wide association study was conducted to evaluate global DNA methylation in a retrospective cohort of 80 mixed subtypes of primary ovarian cancers and 30 patients with high-grade serous ovarian carcinoma (HGSOC). Three demethylating agents, azacytidine, decitabine, and thioguanine, were tested their anti-cancer and anti-chemoresistant effects on HGSOC cells.

Results: Global DNA hypermethylation was significantly associated with high-grade tumors, platinum resistance, and poor prognosis. We determined that 9313 differentially methylated probes (DMPs) were enriched in their relative gene regions of 4938 genes involved in small GTPases and were significantly correlated with the PI3K-AKT, MAPK, RAS, and WNT oncogenic pathways. On the other hand, global DNA hypermethylation was preferentially associated with recurrent HGSOC. A total of 2969 DMPs corresponding to 1471 genes were involved in olfactory transduction, and calcium and CAMP signaling. Co-treatment with demethylating agents showed significant growth retardation in ovarian cancer cells through differential inductions, such as cell apoptosis by azacytidine or G2/M cell cycle arrest by decitabine and thioguanine. Notably, azacytidine and decitabine, though not thioguanine, synergistically enhanced cisplatin-mediated cytotoxicity in HGSOC cells.
\end{abstract}

Conclusions: This study demonstrates the significant association of global hypermethylation with poor prognosis and drug resistance in high-grade EOC and highlights the potential of demethylating agents in cancer treatment.

Keywords: Ovarian cancer, Methylome profiling, Epigenetic, Demethylating agents, Tumor grading

*Correspondence: dwchan@hku.hk; hysngan@hku.hk

1 Department of Obstetrics and Gynaecology, L747 Laboratory Block, LKS Faculty of Medicine, 21 Sassoon Road, Pokfulam, Hong Kong, SAR, People's Republic of China

Full list of author information is available at the end of the article

\section{Background}

Epithelial ovarian cancer (EOC) is one of the most lethal gynecological malignancies affecting women worldwide [1]. The high mortality rate of this disease is due to its poor prognosis and lack of reliable biomarkers, which result in the majority of EOC patients being diagnosed 
at an advanced stage accompanied by metastasis, chemoresistance, and high recurrence $[1,2]$. EOC is a highly heterogeneous disease and consists of at least four subtypes, namely serous, clear cell, endometrioid, and mucinous, based on histopathological differences among the carcinomas [3]. Among these tumor subtypes, 70\% of EOC is the serous subtype followed by the endometrioid and clear cell subtypes, which account for $11-26 \%$, and the mucinous subtype, which accounts for approximately $3 \%[3,4]$. Both serous and endometrioid subtypes can be classified into high-grade (HG) and low-grade (LG) EOCs [5]. Accordingly, EOC with different histological subtypes and tumor grades exhibits unique genetic mutational status, expression profiling signatures, tumorigenic properties, drug resistance, and clinical characteristics [5-7]. However, the current treatment protocols are not subtype- and/or tumor grade-specific, leading to an insufficient curative rate in this disease. Therefore, it is urgently important to better characterize the impact of genetic/epigenetic events involved in tumor heterogeneity, which may pave the way for personalized therapy.

Accumulating evidence has shown that both genetic and epigenetic alterations cooperatively drive cancer development and progression [8,9]. However, previous studies have largely investigated genetic alterations in human cancers, while the influence of epigenetic modifications on cancer progression and development is comparatively less heavily researched [10]. Rigorous genomic evidence indicates that genetic alterations, such as somatic mutations, are stable in tumor development $[8,9]$. In contrast, dynamic epigenetic changes are stimulated by endogenous or environmental factors [11, 12], indicating that the cancer epigenome is another important modulator mediating metastatic progression and the development of human cancers. DNA methylation is the most heavily studied epigenetic mechanism regulating gene expression in mammalian cells [13]. Hypermethylation of $\mathrm{CpG}$ islands on promoter regions leads to gene silencing, especially tumor suppressor genes (TSGs) [14]. On the other hand, global hypomethylation is also involved in oncogenesis [15]. The DNA methylation pattern is heritable but reversible and can be modulated by physiological and environmental stimuli $[16,17]$. This phenomenon supports that the deregulated DNA methylation pattern is associated with cancer progression and tumor heterogeneity [18]. Hence, DNA methylationbased profiling is an additional parameter to consider for classifying tumor subtypes and achieving improved prognosis and treatment of human cancers $[19,20]$.

In this study, we performed an epigenome-wide association study (EWAS) of a retrospective cohort of 80 patients with mixed tumor subtypes and 30 HGSOC of primary ovarian cancers by utilizing the Illumina
Infinium ${ }^{\circledR}$ MethylationEPIC BeadChip system (850 k) as the platform to interrogate over 850,000 CpG sites in the human genome. DNA methylation profiling analysis combined with clinical classifications provided a novel and in-depth understanding of the pathogenesis of EOC and the methylation signatures that may be employed in the diagnosis and treatment of this cancer.

\section{Materials and methods}

\section{Study population and clinical samples}

Eighty patients of epithelial ovarian cancer (EOC) with mixed tumor subtypes, stages, and grades, and 30 patients of high-grade serous subtype (HGSOC) who either survived or experienced disease recurrence after receiving first-line chemotherapy using a combination of carboplatin/paclitaxel) within 1996-2017 were randomly selected from our tissue bank in the Department of Obstetrics and Gynecology, the University of Hong Kong. All tumor tissues were collected in Queen Mary Hospital, Hong Kong, and were stored in liquid nitrogen with patient consent and prior approval of the Institutional Review Board of the University of Hong Kong/Hospital Authority Hong Kong West Cluster (HKU/HA HKW IRS) (IRS Reference Number: UW 11-298).

\section{Histological assessment and genomic DNA isolation}

Frozen cryosections were prepared from the optimal cutting temperature (OCT) frozen blocks of the frozen tumor tissue samples. Histological assessment was subsequently performed by pathologists who conducted morphological diagnosis of tumor cell percentages on cryosections of the OCT frozen blocks that were stained with hematoxylin and eosin (H\&E). Total genomic DNA was extracted from tumor tissues with $>70 \%$ tumor cell content using a QIAamp ${ }^{\circledR}$ Fast DNA Tissue Kit (cat. no. 51404) (QIAGEN) according to the manufacturer's instructions. In general, frozen tissue samples suspended in lysis buffer containing proteinase $\mathrm{K}$ and RNase A were homogenized with a tissue lyser at approximately $45 \mathrm{~Hz}$ for $2 \mathrm{~min}$. The lysates were subsequently applied to the QIAamp Mini Spin Column, and the DNA bond was washed and eluted in nuclease-free water.

\section{Illumina $850 \mathrm{~K}$ methylation assay}

Whole-genome DNA methylation analysis was performed by the Centre for PanorOmic Sciences (CPOS), LKS Faculty of Medicine, the University of Hong Kong, using the Infinium MethylationEPIC microarray covering over 850,000 CpGs (Infinium MethylationEPIC BeadChip Kit, WG-317-1001). In brief, bisulfite conversion was performed on the DNA samples with a Zymo EZ DNA Methylation kit (D5001-Ver.1.2.6) according to the manufacturer's instructions and using $500 \mathrm{ng}$ DNA as 
input. The DNA samples were subsequently denatured, amplified, fragmented enzymatically, and hybridized to Infinium beadchips. The Infinium HD Assay Methylation Protocol Guide [15019519 v06] was followed with the following settings: the incubation time for whole genome amplification was $22 \mathrm{~h} 15 \mathrm{~min}(20-24 \mathrm{~h}$ in the protocol), and the hybridization time was $16 \mathrm{~h} 30 \mathrm{~min}(16-24 \mathrm{~h}$ in the protocol). After hybridization, the beadchips were washed to remove unhybridized and nonspecifically hybridized DNA and were subjected to allele-specific single-base extension and staining thereafter. The beadchips were imaged on Illumina iScan System. Data files were subjected to QC via GenomeStudio Version 2011.1.

\section{Data preprocessing and differential methylation analysis}

Illumina intensity data files (.idat) from the chip were further processed by the R/Bioconductor (version 3.6.1) package ChAMP (version 2.8.6). Probes with a $P$ value $>0.01$ and those located on the $X$ and $Y$ chromosomes were filtered out. In addition, any probes with $<3$ beads in at least $5 \%$ of samples per probe, non-CpG probes, SNP-related probes, and all multi-hit probes were filtered out. The beta-mixture quantile (BMIQ) method was used for type-II probe normalization. Differentially methylated positions (DMPs) between each group were detected with ChAMP. DMP function using ChAMP package. We identified differentially methylated probes (DMPs) at the significance of Benjamini-Hochberg correction with adjusted $P<0.05$ with at least a $20 \%$ difference in the methylation level between each compared group. Additionally, differentially methylated regions (DMRs) within gene promoters or first exons were defined by ChAMP. DMR function from the ChAMP package. Benjamini-Hochberg correction with adjusted $P<0.05$ was used to estimate significant DMRs containing at least $7 \mathrm{CpG}$ probes, and two consecutive locations in the DMR are separated by less than some distance of $300 \mathrm{bp}$.

\section{Functional annotation and pathway enrichment analysis of genes with DMPs}

The gene symbols of genes with differentially methylated probes (DMPs) were converted into the Ensembl Gene ID by org.Hs.eg.db-Bioconductor in R. Gene Ontology (GO) and KEGG enrichment analyses were performed by using the clusterProfiler package in $\mathrm{R}$.

\section{Patient survival analysis}

Kaplan-Meier survival was performed using the R package 'survival'[21]. Significance in overall or disease-free survival was calculated using the log-rank test. Cox proportional hazards regression was performed using the function coxph () from the R package 'survival'[22, 23].

\section{Cell culture}

Ovarian cancer (OvCa) cells (source: female) from the ES2, PEO1, and PEO4 cell lines were purchased from American Type Culture Collection (ATCC). OVCA433 cells (source: female) were provided by Prof. G.S.W. Tsao, School of Biomedical Sciences, the University of Hong Kong. A2780s and A2780cp cells were provided by Prof. Benjamin Tsang, University of Ottawa, Canada. A2780s and A2780cp cells (source: female) were two cell lines with similar origins, while A2780cp exhibited promising cisplatin resistance. PEO1 and PEO4 were another pair, with $\mathrm{PEO} 4$ showing enhanced cisplatin resistance. OVCA433 and ES2 cells were cultured in Dulbecco's modified Eagle's medium (DMEM) with 10\% (v/v) fetal bovine serum (FBS) and $1 \%(\mathrm{v} / \mathrm{v})$ penicillin-streptomycin (PS). A2780s, A2780cp, PEO1, and PEO4 cells were cultured in Roswell Park Memorial Institute (RPMI) 1640 medium with $10 \%(\mathrm{v} / \mathrm{v})$ FBS and $1 \%(\mathrm{v} / \mathrm{v})$ PS. Cells were cultured in a humidified incubator with $5 \%$ carbon dioxide $\left(\mathrm{CO}_{2}\right)$ at $37^{\circ} \mathrm{C}$.

\section{D cell viability assay (XTT assay)}

Ovarian cancer cells were seeded onto 96-well clear flatbottom microplates at a density of $1 \times 10^{4}$ cells $/ \mathrm{mL}$ in $100 \mu \mathrm{L}$ cell culture medium. The cells were treated with cisplatin, azacytidine, decitabine, or thioguanine at different concentrations in serial dilutions for $48 \mathrm{~h}$. Cell viability was determined using the XTT assay. The culture medium was removed, and XTT reagent $(150 \mu \mathrm{L})$ was added to each well. The plate was incubated in an incubator for $4 \mathrm{~h}$. The absorbance at $492 \mathrm{~nm}$, which represented cell viability, was measured by a microplate reader.

3D cell viability assay (CellTiter-Glo ${ }^{\circledR} 3 \mathrm{D}$ cell viability assay) Ovarian cancer cells were resuspended in serum-free DMEM/F12 (1:1) medium supplemented with $10 \mathrm{ng} / \mathrm{mL}$ human basic fibroblast growth factor (hbFGF), $20 \mathrm{ng} /$ $\mathrm{mL}$ human epidermic growth factor (hEGF), and $5 \mu \mathrm{g} /$ $\mathrm{mL}$ insulin at a cell density of $1 \times 10^{4}$ cells $/ \mathrm{mL}$. The cell suspension (1000 cells in $100 \mu \mathrm{L}$ medium) was added to ultra-low attachment Nunclon ${ }^{\mathrm{TM}}$ Sphera $^{\mathrm{TM}}$ 96U microplates (Thermo) and incubated for 5 days to allow spheroid formation. The spheroids were treated with cisplatin (M2223, AbMole BioScience, Houston, TX, USA) at different concentrations in serial dilutions $(0$ to $80 \mu \mathrm{M})$ and cotreated with azacytidine (M2291, AbMole BioScience), decitabine (M2052, AbMole BioScience), or thioguanine (M3258, AbMole BioScience) at various concentrations in serial dilutions $(0$ to $160 \mu \mathrm{M})$ for $72 \mathrm{~h}$. Spheroid viability after drug treatment was determined using a CellTiter-Glo ${ }^{\circledR}$ 3D cell viability assay. Equal volumes of CellTiter-Glo ${ }^{\circledR}$ 3D Reagent were added to each 


\begin{abstract}
(See figure on next page.)
Fig. 1 Higher global DNA methylation levels are frequently found in high-grade EOC. a Heatmap visualization constructed using k-means clustering of differential methylation positions (DMPs:14120) (5\% false discovery rate) with at least a $20 \%$ difference in the methylation level between low- and high-grade across 80 EOC. $\mathbf{b}$ Boxplot visualization of comparison of DMP methylation levels of low- and high-grade EOC. The median DMP methylation levels were 0.445 and 0.637 in the low- and high-grade groups, respectively. $P$ value (**** $P<0.0001)$ was computed by the Wilcoxon test. c Boxplot visualization of comparisons of DMP methylation levels of low- and high-grade DMPs, as well as the associated hypermethylated and hypomethylated DMPs. The median value of each group is shown as a line of each box and number. $P$ value (****P<0.0001) was computed by the Wilcoxon test. $\mathbf{d}$ Manhattan plot of differentially methylated positions, where each point represents the observed $\log 10$-adjusted $P$ value at each CpG site. A total of the top 16 significant DMPs (annotated to 17 DMP-associated genes) (adjusted $P<5^{*} 10^{-11}$ ) found on the relevant gene promoter regions were marked. e Volcano plot showing the distribution of 11,986 hypermethylated DMPs (red) and 2134 hypomethylated DMPs (blue) with FDR-adjusted $P<0.05$ and difference in $\beta$ value ( $|\log F C|>0.2)$. Dashed lines represent cutoffs for the significant DMP-associated genes. The most differentially regulated genes of hypomethylated and hypermethylated sites are marked. (F) DMR at the promoter regions of CLDN16 and HPS4. Results are plotted in comparison of DNA methylation levels of each CpG site located in the promoter regions CLDN16 and HPS4 in low- and high-grade EOC
\end{abstract}

well. The spheroids were dissociated by being pipetted up and down multiple times. The reaction mixture $(100 \mu \mathrm{L})$ was transferred to 96-well white flat-bottom microplates and incubated at room temperature for $30 \mathrm{~min}$. The luminescence representing the viability of the spheroids was measured by a Glomax ${ }^{\circledR} 96$ Microplate Luminometer. The synergistic or antagonistic effect of cisplatin with azacytidine, decitabine, and thioguanine was analyzed using Combenefit software.

\section{Annexin V-PI staining}

OvCa cells were seeded into 6-well tissue culture plates at $2 \times 10^{5}$ cells/well overnight. Next, the cells were treated with different concentrations of cisplatin, azacytidine, decitabine, and thioguanine for $48 \mathrm{~h}$. Cells were trypsinized and washed with PBS three times. The cells were stained with $100 \mu \mathrm{L}$ staining solution with Annexin V-Fluorescein and propidium iodide (PI) for $15 \mathrm{~min}$ in the dark. After staining, $400 \mu \mathrm{L}$ staining buffer was added to the cells. Flow cytometry was performed using a CytoFLEX flow cytometer (Beckman-Coulter). The data were analyzed using CytExpert Software (Beckman-Coulter).

\section{Cell cycle analysis}

Ovarian cancer (OvCa) cells were seeded on $90-\mathrm{mm}$ culture dishes at $5 \times 10^{5}$ cells $/$ dish. The cells were starved in serum-free RPMI or DMEM overnight followed by treatment with different concentrations of cisplatin, azacytidine, decitabine, and thioguanine for $48 \mathrm{~h}$. Cells were trypsinized, washed with PBS, and fixed in $70 \%$ ethanol at $-20{ }^{\circ} \mathrm{C}$ for $2 \mathrm{~h}$. The cells were centrifuged at $800 \mathrm{~g}$ for $5 \mathrm{~min}$ and washed with PBS three times. The cells were stained with $250 \mu \mathrm{L}$ of $50 \mu \mathrm{g} / \mathrm{mL}$ PI in PBS for $15 \mathrm{~min}$ in the dark. After staining, $750 \mu \mathrm{L}$ PBS was added to the cells. Flow cytometry was performed using a CytoFLEX flow cytometer. The proportion of cells in each phase of the cell cycle was determined using FlowJo software.

\section{Statistical analysis}

Statistical analysis of global DNA methylation variation was performed using unsupervised hierarchical clustering analysis with Euclidean distances. Wilcoxon test was performed to test for significant differences in DMP methylation level between the groups. Survival curves were plotted according to the Kaplan-Meier method. The statistical significance between curves was tested using the log-rank test. On the other hand, all functional assays were performed by GraphPad Prism 6.0 (San Diego, CA, USA). All data were obtained from at least three independent experiments, each performed in triplicate. Data were analyzed by unpaired t-test, and data were expressed as the mean \pm S.E.M. of at least three independent tests. $P$ values $<0.05$ were considered to be significant.

\section{Results}

Elevated epigenome-wide DNA methylation status is associated with high-grade EOC

This study performed EWAS using MethylationEPIC BeadChip (Illumina) to quantitatively interrogate over 850,000 CpG positions across the genome in 80 tumor tissues of mixed and pretreated EOC. The differential methylation analysis demonstrated that there was no significant correlation with tumor stages or histological subtypes of these 80 samples. Notably, there were 14,120 differentially methylated probes (DMPs) (5\% false discovery rate) with at least a $20 \%$ difference in the methylation level between low-grade and high-grade EOC ( $\mid \Delta$ beta $\mid>0.2$ ) (Fig. 1a). Among 80 samples, there were three samples of grade 1, 26 samples of grade 2, 49 samples of grade 3, one sample was denoted by high grade, and one unknown tumor grading case. To improve the analysis, we omitted the unknown tumor grading case but categorized grades 1 and 2 cases as "lowgrade" EOC and classified grade 3 as "high-grade" EOC. EWAS analysis demonstrated that a significantly higher content of global DNA methylation could be found in 
A

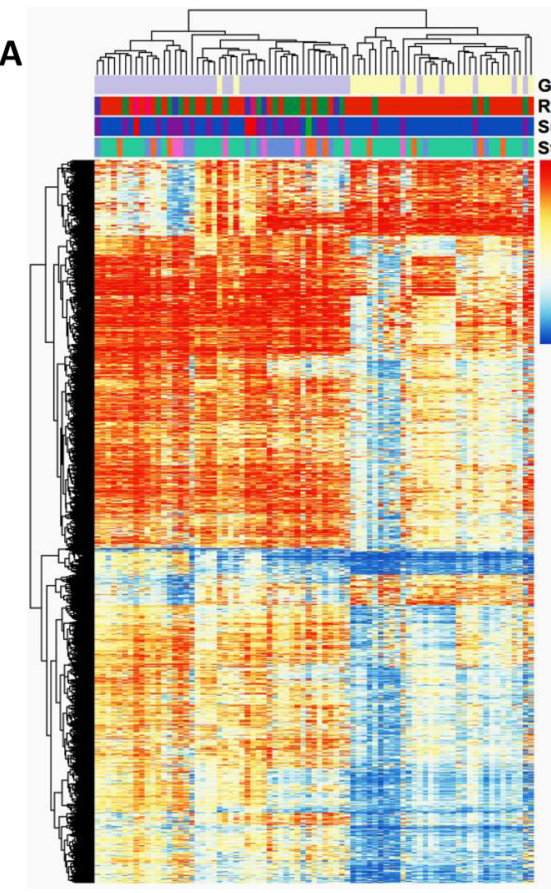

GRADE Recurrence Stage

0.8 Low

0.6 High

0.4 Recurrence

0.2 recurrence

disease progression

disease progression

Survivial.status

alive without specified

alive without disease

alive

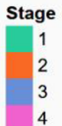

B
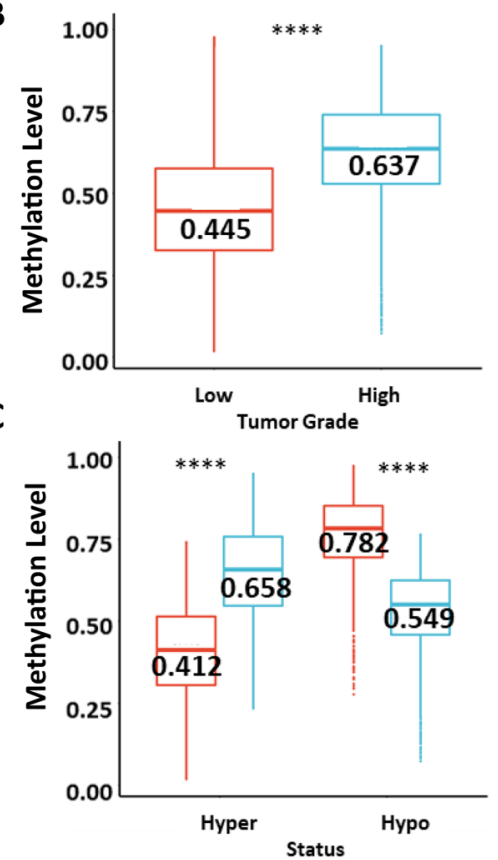

D

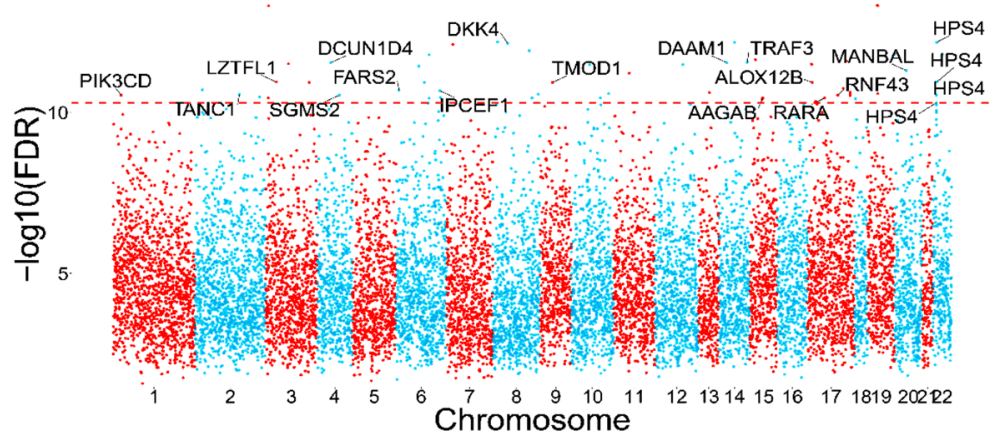

E

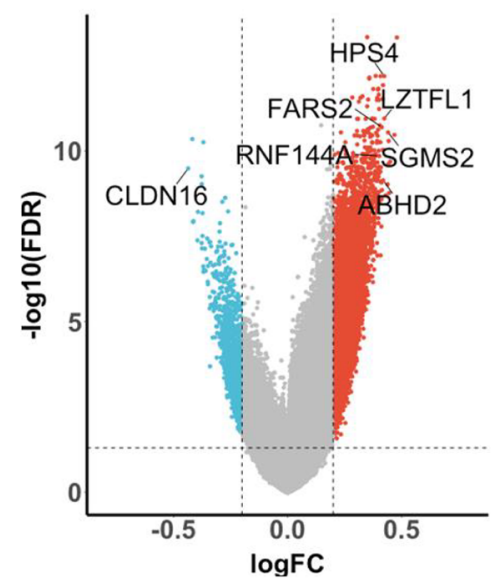

$\mathbf{F}$

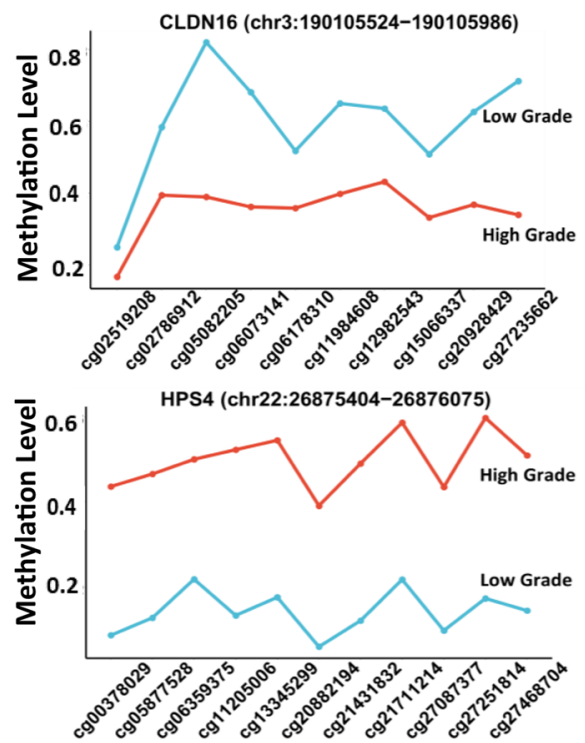

Fig. 1 (See legend on previous page.) 
(See figure on next page.)

Fig. 2 Gene ontology analyses of the differentially methylated DMPs between high- and low-grade EOC. a Gene ontology (GO) enrichment analysis of 4938 DMPs-associated genes enriched by 8064 hypermethylated and 1249 hypomethylated DMPs. The vertical axis represents the GO category, and the horizontal axis represents the gene count, and the color represents the p-adjusted value. b Kyoto Encyclopedia of Genes and Genomes (KEGG) signaling pathway enrichment of differentially methylated genes analyzed by the clusterProfiler package in R. The circle size represents gene numbers, and the color represents the $p$-adjusted value

high-grade $\mathrm{EOC}$ (median value $=0.637)$ than in low-grade EOC (median value $=0.445$ ) (Fig. 1, a, b). Among 14,120 DMPs, 11,986 (84.89\%) were hypermethylated CpG sites associated with high-grade EOC (median value $=0.658$ ), which was significantly higher than that associated with low-grade EOC (median value $=0.412$ ) (Fig. 1c). On the other hand, 2134 out of 14,120 (15.58\%) DMPs were hypomethylated $\mathrm{CpG}$ sites, which were strongly associated with low-grade EOC (median value $=0.782$ ) and were significantly higher than those associated with high-grade EOC (median value $=0.549$ ) (Fig. 1c). These findings indicate that the global DNA hypermethylation level is higher in high-grade EOC compared with low-grade EOC.

Given that global DNA hypermethylation has occurred in high-grade EOC, we were interested in analyzing the distribution of DMPs with biologically relevant on the 22 autosomal chromosomes. Our analysis indicated that the top 17 DMP-associated genes (promoter regions) were found on chromosomes 1-4, $68-9,14,1517,20$, and 22 from 14,120 DMPs at a false discovery rate (FDR) threshold $\left(P<5^{*} 10^{-11}\right)$ (Fig. 1d) (Additional file 1: Table S1). After further analysis by screening the hypermethylated or hypomethylated $\mathrm{CpG}$ sites within the promoter regions from the transcription start site (TSS) [24], we identified six genes, CLDN16 (hypomethylated), HPS4, LZTFL1, FARS2, SGMS2, ABHD2 and RNF144A (hypermethylated), that were potentially dysregulated and could be a novel DNA methylation signature in the classification of tumor grading (Fig. 1e). Kaplan-Meier analysis of the above-mentioned methylation signatures indicated that the hypomethylated CLDN16 locus was positively correlated with poor overall survival (OS) in EOC (Additional file 5: Fig. S1). In contrast, the hypermethylated loci of LZTFL1, FARS2, SGMS2, ABHD2, RNF144A and HPS4 were positively correlated with the poor overall survival (OS) of EOC patients (Additional file 5: Fig. S1). Indeed, the hypomethylated gene promoter regions of $C L D N 16$ or the hypermethylated promoter regions of LZTFL1, FARS2, SGMS2, ABHD2, RNF144A and HPS4 were associated with high-grade, advanced stages (stages 3 and 4), clear cell and serous subtypes, recurrence, and high mortality rates (Additional file 6: Fig. S2). Notably, computational analysis of differentially methylated regions (DMRs) demonstrated that the levels of 10 hypomethylated CPG sites of the CLDN16 promoter region on chr3:190105524-190105986 were significantly higher in low-grade EOC, whereas the overall levels of 11 hypermethylated CpG sites of the HPS4 promoter region on chr22:26875404-26876075 were significantly higher in high-grade EOC (Fig. 1f). These findings indicated that the methylation status of the above-mentioned DMP-associated genes could be used as DNA methylation signatures to predict and classify aggressiveness and highor low-grade EOC.

\section{Identification of signaling pathways relevant to the epigenetic signature of high-grade EOC}

To understand the overall functional relevance of 9313 DMPs (enriched in gene regions including promoter, gene body, 3'UTR), including 4938 genes, we performed gene ontology (GO) enrichment analysis in two annotations: biological processes and molecular functions. The results indicated that the most significantly enriched GO terms were small GTPase for signal transduction followed by RAS protein signal transduction and cell-cell signaling by WNT (Fig. 2a). In keeping with these results, among all differentially methylated genes in modulating small GTPase activities, the highest differential DMPs are enriched in their relative gene regions (promoter, gene body, $3^{\prime} \mathrm{UTR}$ ) in relation to TIMP2, $R H O H$, EphB2, and $A R R B 1$, which have been shown to be tumor suppressors and may be inhibitors of small GTPases (Additional file 2: Table S2) [25-27]. Similarly, KEGG (Kyoto Encyclopedia of Genes and Genomes) pathway analysis showed that these genes were enriched in well-known cancer pathways, such as the PI3K-AKT, MAPK, RAS, and WNT cancer signaling pathways (Fig. 2b). This enrichment was evidenced by key hypermethylated TSGs, such as PTEN and PIK3R1 of the PI3K-AKT signaling pathway [28, 29], EFNA3 and NF1 of the MAPK signaling pathway [30,31], ABL1 and PAK1 of the RAS signaling pathway [32], and PRICKLE1 and $D A A M 1$ of the WNT signaling pathway [33], and these key TSGs were significantly correlated with poor OS in EOC (Additional file 7: Fig. S3, Additional file 3: Table S3). Taken together, these results indicate that hypermethylation of TSGs confers aberrant activation of oncogenic pathways involved in the aggressiveness and poor prognosis of highgrade EOC.

\section{Global DNA methylation increases the recurrence risk and mortality of EOC}

High-grade EOC is usually characterized by high aggressiveness and metastatic potential [12, 34]. In this study, 
A

\section{Biological Process}

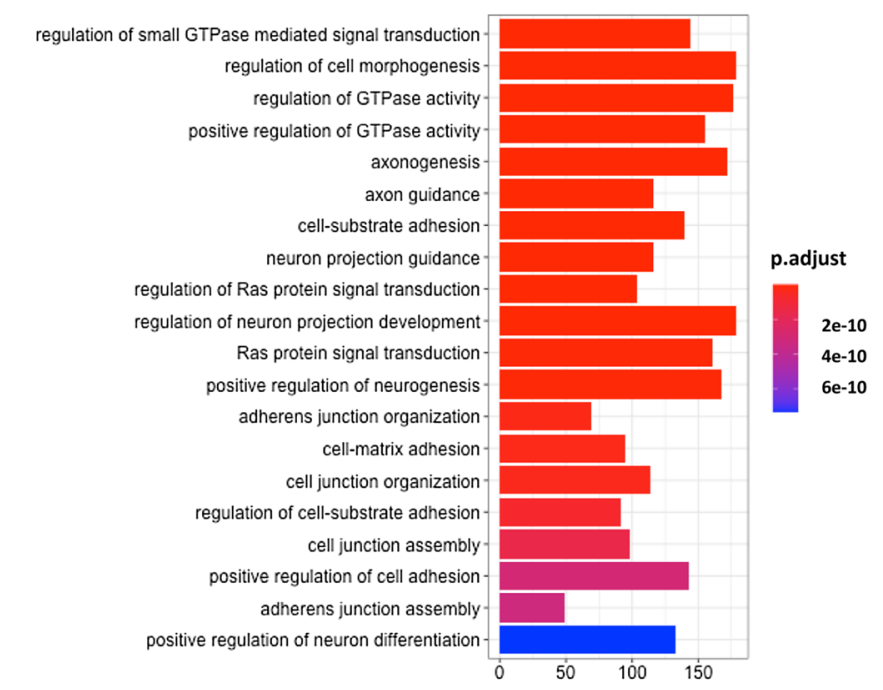

\section{Molecular Functions}

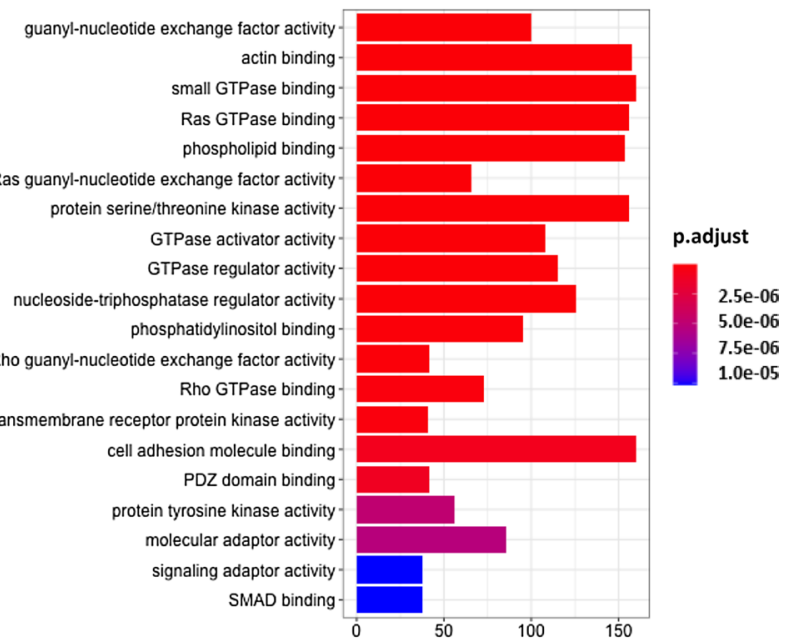

B

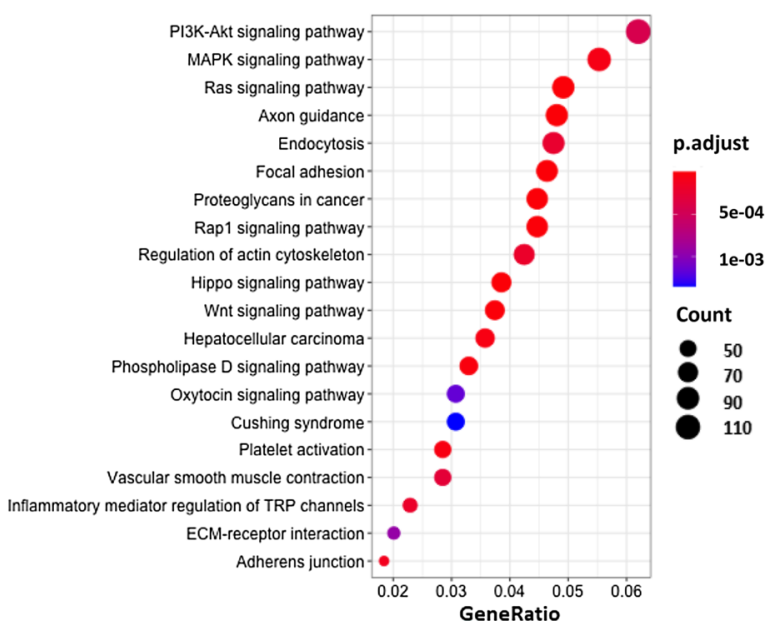

Fig. 2 (See legend on previous page.) 
(See figure on next page.)

Fig. 3 EOC with higher global DNA methylation levels has higher recurrence and poor overall survival (OS). a Heatmap visualization constructed using k-means clustering of differential methylation positions (DMPs:301) (5\% false discovery rate) with at least a $20 \%$ difference in the methylation level between "alive without disease" and "alive with disease plus died" groups across 80 EOC cases. b Volcano plot showing the distribution of 180 hypermethylated DMPs (red) and 121 hypomethylated DMPs (blue) with FDR-adjusted $P<0.05$ and difference in $\beta$ value ( $\mid$ logFC $>0.2)$. Dashed lines represent cutoffs for significance. The most differentially regulated genes of hypomethylated and hypermethylated sites are marked. Kaplan-Meier estimate of OS using the methylation signature of genes c CLDN16 and PIK3C2G) and $\mathbf{d}$ WWP1, FNDC3B, DISP1, NRIP1, PDE4D and CLDN12

although our EWAS analysis could not show any significant correlation of DMPs with tumor stages, 301 DMPs are associated with a reciprocal relationship between tumor recurrence and overall survival (OS) in 80 EOC samples (Fig. 3a). Heatmap analysis of the methylation values of significant DMPs showed that EOC patients with recurrence were associated with short-term survival, and the reverse was also observed (Fig. 3a). Of 200 DMPs, we found that 177 genes had either hypomethylated or hypermethylated DMPs enriched in their relative promoter regions. The most highly differentially methylated genes enriched by DNA hypomethylated DMPs were CLDN16 and PIK3C2G, while those enriched by the DNA hypermethylated DMPs were WWP1, DISP1, FNDC3B, NRIP1, FDE4D, and CLDN12 (Fig. 3b). Notably, these hypomethylated and hypermethylated genes were correlated with shorter overall survival (OS) in EOC (Fig. 3c, d). These findings suggest that the DNA methylation signatures could be used to predict the disease recurrence and survival status of EOC.

\section{DNA methylation is associated with platinum resistance}

Emerging evidence has shown that DNA methylation is involved in chemoresistance in solid cancers [35], and acquired platinum resistance is commonly observed in high-grade serous subtype ovarian cancer (HGSOC) [36]. Our EWAS indicated that 5844 DMPs could significantly show DNA methylation differences in 30 HGSOC samples (Fig. 4a). We found that 10 clinical cases belonged to the platinum-sensitive group with lower global methylation levels. Of these 10 clinical cases, 6/10 (60\%) showed no recurrence within 5 years (Fig. 4a). On the other hand, the other 20 clinical cases were in the platinum-resistant group and exhibited higher global methylation levels. However, only 1 case $(1 / 20,5 \%)$ showed no recurrence within 5 years after receiving taxane-/ platinum-based therapy as a first-line treatment (Fig. 4a). In addition, the platinum-resistant group exhibited a significantly higher content of global DNA methylation (median value $=0.692$ ) than the platinum-sensitive group (median value $=0.373$ ) (Fig. 4a, b). Further analysis showed that 2969 DMPs corresponding to 1471 differentially methylated genes were associated with significant DNA methylation. Among these differentially methylated genes, the top $20 \mathrm{DMP}$-associated genes (promoter regions) were found on chromosomes 1-3, 5, 11-12,
14-15, 17 and 19 at a false discovery rate (FDR) threshold $\left(P<5^{*} 10^{-3}\right)$ (Fig. 4c) (Additional file 4: Table S4). Notably, the majority of DMPs were significantly associated with hypermethylated genes, including OR51L1, OR51I1, OR51F1, OR51B6, HBBP1, TMEM200A, and $D L G 2$ (Fig. 4d). Importantly, the DNA methylation levels of DMPs associated with these hypermethylated genes were significantly higher in the platinum-resistant group (Fig. 4e). GO enrichment analysis revealed that the most significantly differentially methylated genes were correlated with sensory perception, chemical stimulus detectors, and ion transmembrane transport regulation in biological functions, as well as ion channel activities in molecular functions (Additional file 8: Fig. S4). Additionally, KEGG enrichment analysis showed that the most enriched pathways included olfactory transduction, neuroactive ligand-receptor interaction, calcium signaling, and cAMP signaling pathways (Fig. 4f). Taken together, the results of our EWAS analysis indicated that HGSOC with higher global DNA methylation is usually involved in different levels of platinum resistance, leading to varying time intervals of recurrence after primary taxane-/ platinum-based therapy.

\section{Demethylating agents could impair the tumor growth of ovarian cancer cells}

High-grade cancers are characterized by a high potential for aggressiveness and acquired drug resistance [37]. Given that high global DNA methylation is associated with high grade and platinum resistance in EOC, the inhibition of overall DNA methylation by demethylating agents could effectively target the aggressiveness and drug resistance of EOC. To this end, we tested three commercially available demethylating reagents, azacytidine [38], decitabine [39] (suppression of DNA methyltransferase and induction of hypomethylation of DNA), and thioguanine (a purine antagonist) [40], on the viability of ovarian cancer cells. We first evaluated the IC50 values of each drug, including cisplatin, a common platinum reagent used in EOC treatment [41].

We tested 6 high-grade ovarian cancer cell lines, specifically OVCA33, PEO1, PEO4, ES-2, and a pair of isogenic ovarian cancer adenocarcinoma cell lines, A2780s (cisplatin-sensitive) and A2780cp (cisplatin-resistant) [42, 43]. The IC50 of cisplatin on these cell lines ranged 


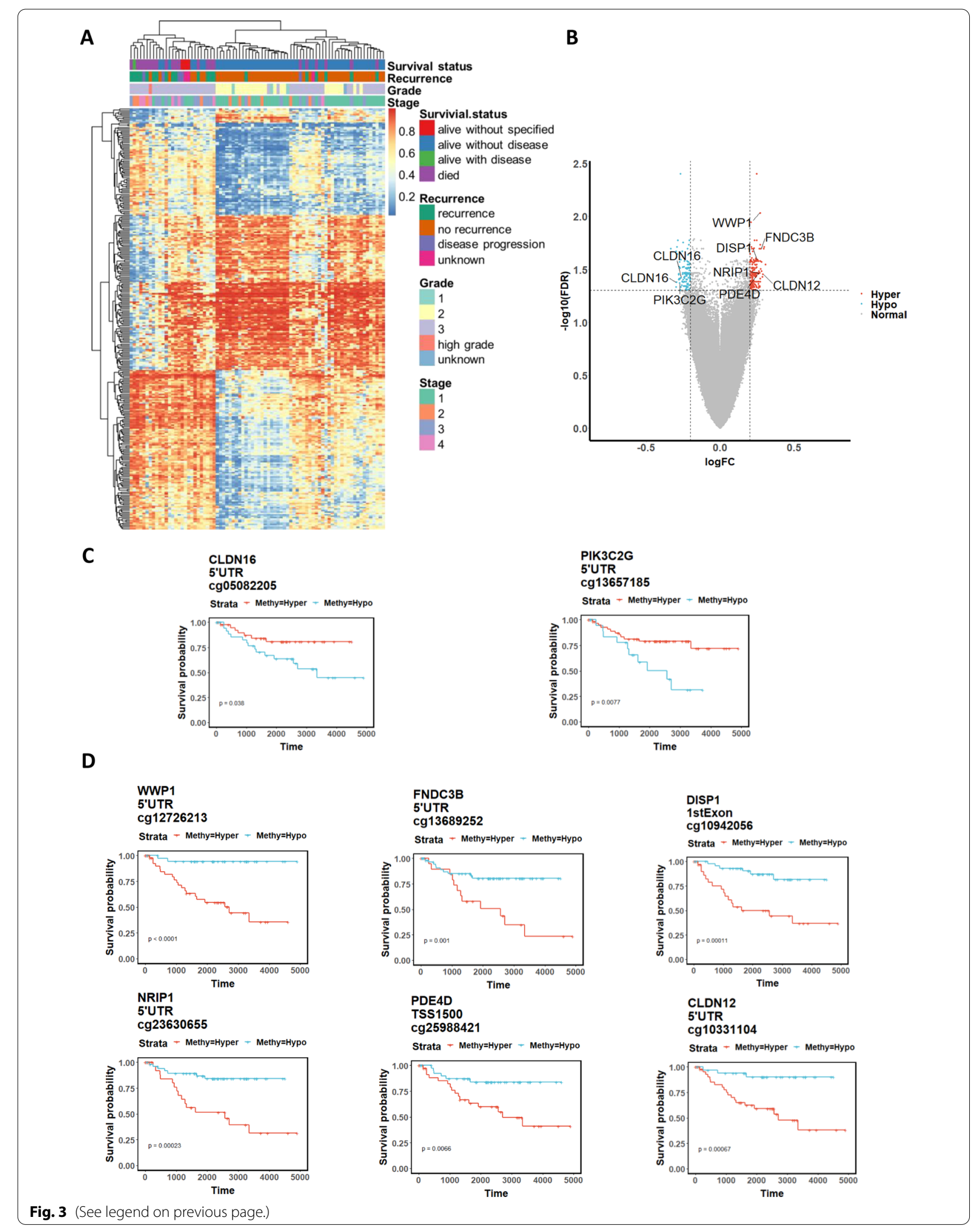




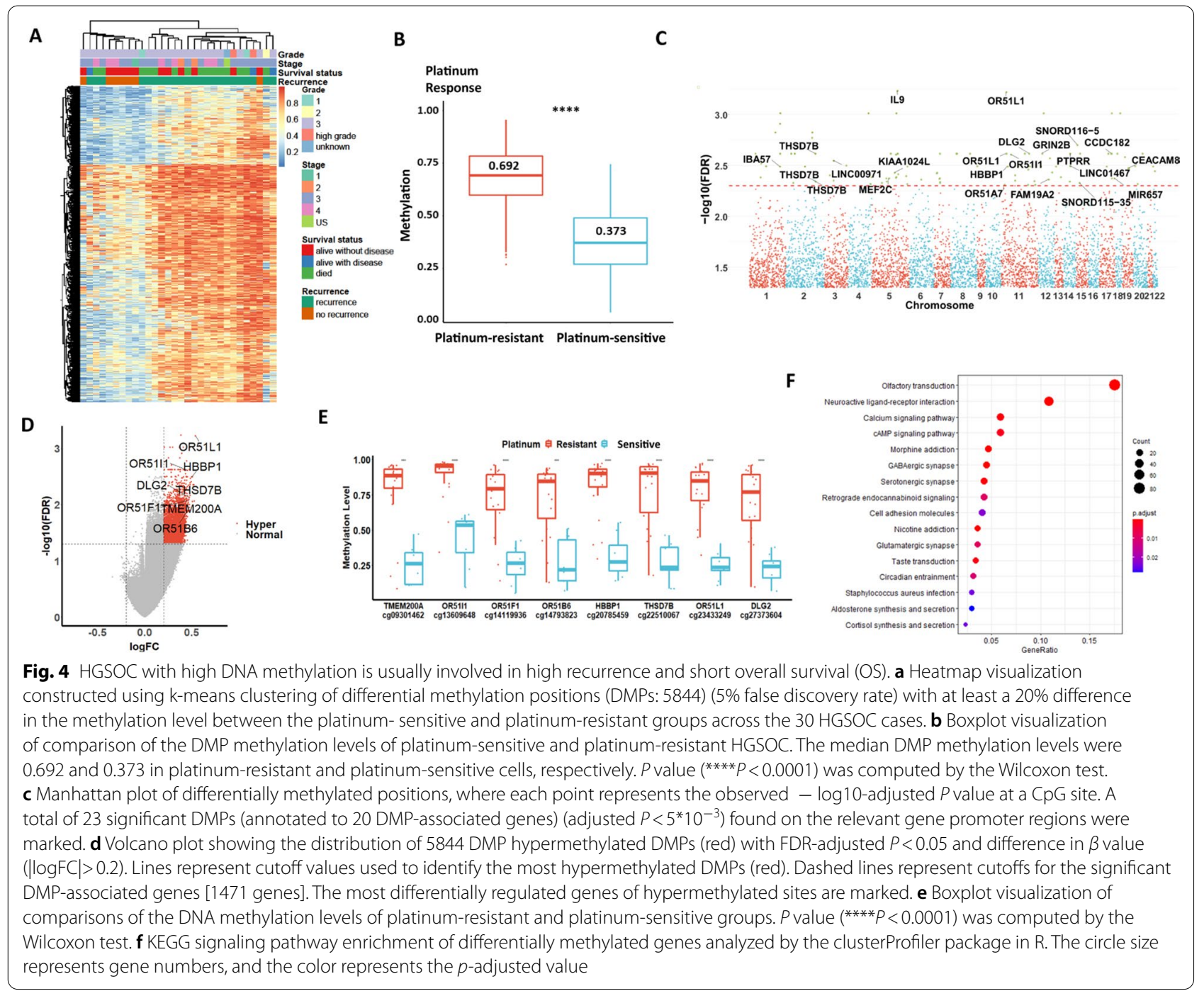

from $1.94 \mu \mathrm{M}$ to $11.95 \mu \mathrm{M}$, with A2780s cells being the most vulnerable to cisplatin cytotoxicity, while A2780cp and OVCA433 cells were considerably more resistant to cisplatin cytotoxicity (Fig. 5a, Additional file 9: Fig. S5A). The IC50 of azacytidine ranged from $1.97 \mu \mathrm{M}$ in A2780s cells to $8.90 \mu \mathrm{M}$ and $9.02 \mu \mathrm{M}$ in OVCA433 and PEO4 cells, respectively (Fig. 5a) (Additional file 9: Fig. S5A). On the other hand, decitabine had mild or little inhibitory effect on all 6 cell lines, with IC50 values exceeding the maximal treatment dose at $80 \mu \mathrm{M}$ (Fig. 5a, Additional file 9: Fig. S5A). Thioguanine performed better than decitabine and caused cytotoxicity in ovarian cancer cells; the IC50 was $1.17 \mu \mathrm{M}$ for OVCA433 cells, which had a higher IC50 value at $20.98 \mu \mathrm{M}$ for A2780cp cells (Fig. 5a, Additional file 9: Fig. S5A).

Next, we determined whether the cytotoxicity of cisplatin and three demethylating agents induced cell apoptosis or retarded cell division. To this end, we focused on
A2780s and A2780cp because of their significant difference in drug responses to cisplatin and other demethylating reagents. By an Annexin V-PI staining assay, the flow cytometry analysis showed that the cisplatin-resistant A2780cp cells showed very little apoptosis until they were treated with $2.5 \mu \mathrm{M}$ cisplatin, while the cisplatinsensitive A2780s cells showed increasing apoptotic cell populations from $2.5,10$ to $40 \mu \mathrm{M}(17.63 \%, 32.61 \%$, and 63.41\%, respectively) (Fig. 5b, Additional file 9: Fig. S5, B and $\mathrm{C}$ ). Azacytidine also induced cell apoptosis in both cell lines, with A2780s cells being more responsive than A2780cp cells (Fig. 5b, Additional file 9: Fig. S5, B and C). Both decitabine and thioguanine had minor or no effect on apoptosis induction in A2780cp and A2780s cells (Fig. 5b, Additional file 9: Fig. S5, B and C).

Cell cycle analysis demonstrated that cisplatin could induce G2/M cell cycle arrest in both A2780cp and A2780s cells (Fig. 5c, Additional file 9: Fig. S5, D and E). 


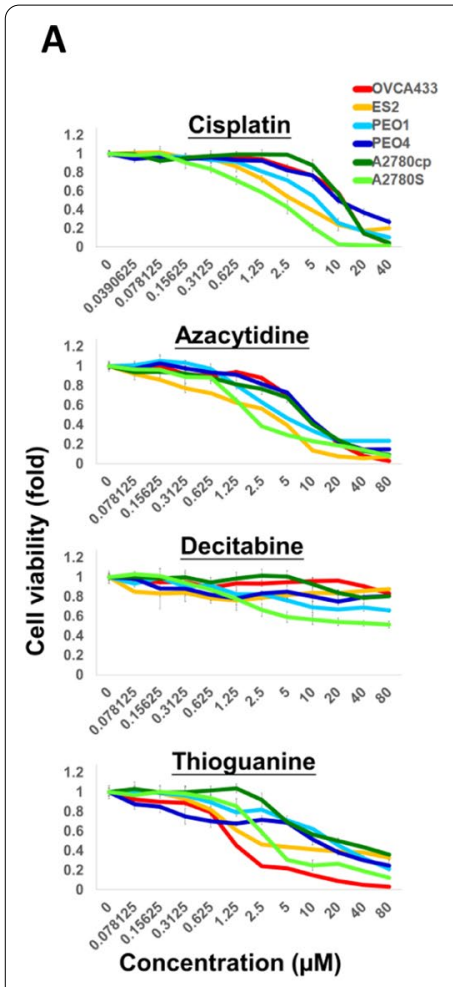

B

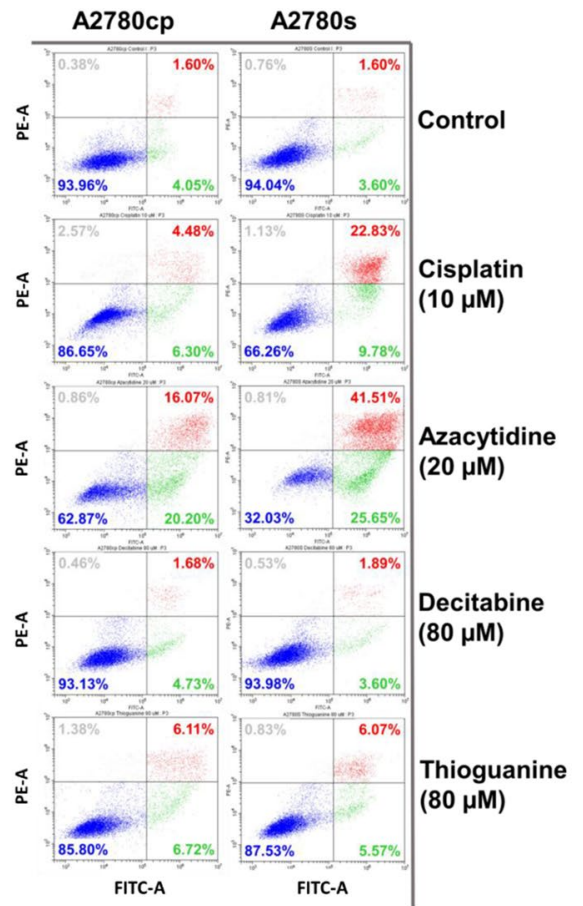

C

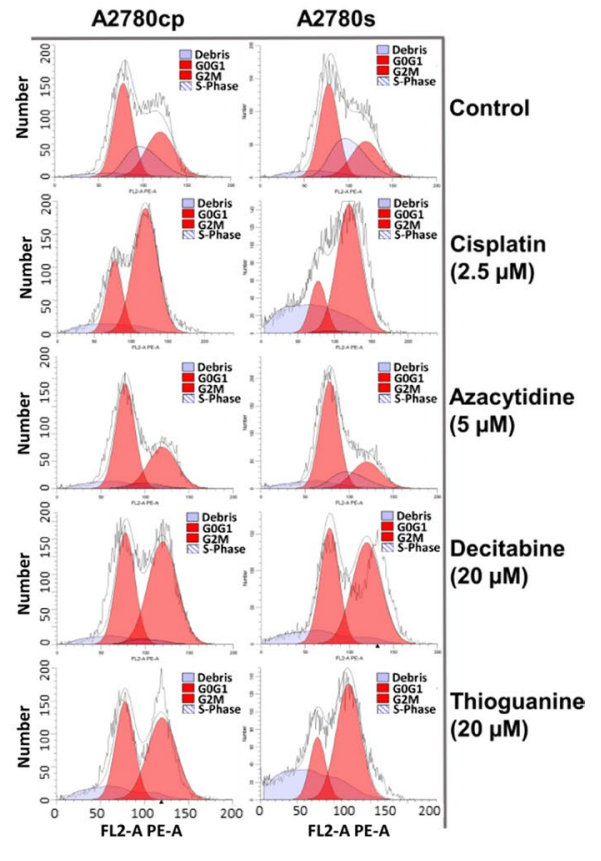

Fig. 5 Cisplatin, azacytidine, decitabine, and thioguanine exerted inhibitory effects on ovarian cancer cell lines in vitro. a XTT assay demonstrated the reduction of cell viability on different ovarian cancer cell lines upon treatment with different concentrations of cisplatin, azacytidine, decitabine, and thioguanine for $48 \mathrm{~h}$. b Flow cytometry on Annexin V-PI staining showed the effect of cisplatin $(10 \mu \mathrm{M})$, azacytidine $(20 \mu \mathrm{M})$, decitabine $(80 \mu \mathrm{M})$ and thioguanine $(80 \mu \mathrm{M})$ on the induction of apoptosis in A2780cp and A2780s cells after a 48-h treatment. Data analysis was performed with CytExpert software. Populations of cells distributed in different quadrants are presented in different colors. c Flow cytometry on PI staining revealed the effect of cisplatin $(2.5 \mu \mathrm{M})$, azacytidine $(5 \mu \mathrm{M})$, decitabine $(20 \mu \mathrm{M})$, and thioguanine $(20 \mu \mathrm{M})$ on interruption of cell cycle progression on A2780cp and A2780s cells after a 48-h treatment. Data analysis was performed with Modfit LT software. Populations of cells distributed in different phases are presented in different shaded peaks. For all, $n=3$ technical replicates per sample. Data are represented as the mean \pm SEM. ${ }^{*} P<0.05$; ${ }^{* *} P<0.01$; ${ }^{* * *} P<0.0001$ (Student's $t$ test)

The cell population of $\mathrm{G} 2 / \mathrm{M}$ phase A2780cp cells was drastically elevated upon treatment with 0 to 2.5 and $10 \mu \mathrm{M}$ cisplatin $(32.01 \%, 72.72 \%$, and $91.57 \%$, respectively) (Fig. 5c, Additional file 9: Fig. S5, D and E). In contrast, lower doses of cisplatin from $0.625-2.5 \mu \mathrm{M}$ were required to cause G2/M cell cycle arrest in A2780s cells by $46.76-78.12 \%$ (Fig. 5c, Additional file 9: Fig. S5, D and E). Upon azacytidine treatment, no notable cell cycle arrest could be detected in either A2780cp or A2780s cells, but a clear cytotoxic effect was observed in both cells with an increased SubG1 cell population upon higher dose treatment (Fig. 5c, Additional file 9: Fig. S5, $\mathrm{D}$ and $\mathrm{E})$, suggesting that azacytidine could cause cell apoptosis. On the other hand, treatment of A2780cp and A2780s cells with decitabine at 0 to $20 \mu \mathrm{M}$ led to an increase in the G2/M phase cell population from 35.03\% to $56.66 \%$ and $28.38 \%$ to $57.41 \%$, respectively, but there was no increase in the SubG1 cell population (Fig. 5c, Additional file 9: Fig. S5, D and E). Similarly, treatment with thioguanine at 0 to $20 \mu \mathrm{M}$ induced $\mathrm{G} 2 / \mathrm{M}$ arrest in A2780cp cells from $35.08 \%$ to $56.29 \%$, but no change was observed in the SubG1 cell population (Fig. 5c, Additional file 9: Fig. S5, D and E), indicating that decitabine and thioguanine could cause cell cycle arrest only. Taken together, the three demethylating reagents examined in this study function differently in ovarian cancer cells. In brief, azacytidine could induce cell apoptosis without any effect on the cell cycle. In contrast, decitabine and thioguanine exhibited slight proapoptotic effects on cells, but they could impair cell division through the induction of G2/M cell cycle arrest.

\section{Suppression of global DNA methylation synergistically enhances platinum cytotoxicity in ovarian cancer cells} Cisplatin is widely used in cancer therapies, including the treatment of ovarian cancers [41]. However, the development of resistance toward cisplatin is a major obstacle facing the treatment of these cancers. Cotreatment of 
(See figure on next page.)

Fig. 6 Differences in the synergistic effects of cisplatin with azacytidine, decitabine, and thioguanine were observed after cotreatment of cisplatin with the three drugs on 3D spheroids of PEO1, PEO4 and OVCA433 cells by CellTiter-Glo ${ }^{\circledR}$ 3D cell viability assay. Analysis of synergism and antagonism was performed by Combenefit analysis software using the HSA model. The viability plots, HSA synergism and antagonism plots and synergy maps are shown for each treatment on the three ovarian 3D spheroids. The regions showing increasing synergism are presented from cyan to blue, while regions showing increasing antagonism are presented from yellow to red. For all, $N=3$ technical replicates per sample. Data are represented as the mean \pm SEM. ${ }^{*} P<0.05 ;{ }^{* *} P<0.01 ;{ }^{* *} P<0.0001$ (Student's $t$ test)

cisplatin with other drugs is an important approach to enhance the efficacy of cisplatin treatment. In this study, we performed cotreatment of cisplatin with azacytidine, decitabine, and thioguanine to evaluate the influence of the three drugs on the antitumor effect of cisplatin on HGSOC cells. Drugs were applied to 3D spheroids of ovarian cancer cells, in which the architectures and microenvironment of 3D culture exhibited higher similarity to solid tumors with layers of cells. Three HGSOC cell lines, PEO1, PEO4, and OVCA433, were chosen for testing $[44,45]$.

The cell viability of PEO1, PEO4, and OVCA433 spheroids upon cotreatment of cisplatin with azacytidine, decitabine, or thioguanine was examined. Combenefit software was used to analyze synergism and antagonism with the HSA synergy score model (Fig. 6). In the HSA synergism plots of cotreatment with cisplatin and azacytidine, clear synergistic zones were available in PEO1, PEO4, and OVCA433 cells, which represented a significant synergistic effect of cisplatin and azacytidine on the suppression of ovarian cancer cell growth (Fig. 6). Under cotreatment with cisplatin and decitabine, larger synergistic zones were formed, indicating that a robust synergistic effect was achieved when using low doses of decitabine for cisplatin treatment (Fig. 6). In contrast, cotreatment with cisplatin and thioguanine led to antagonistic effects on PEO1 and PEO4, indicating that thioguanine could not cause a synergistic effect on cisplatin cytotoxicity in ovarian cancer cells (Fig. 6). These findings suggest that only azacytidine and decitabine could synergistically enhance the cytotoxic effect of cisplatin, and thioguanine does not appear to influence the antitumor effect, especially in combination with cisplatin in ovarian cancer chemotherapy.

\section{Discussion}

The obstacles facing the clinical management of epithelial ovarian cancer (EOC) are the lack of reliable biomarkers for early diagnosis, the development of acquired drug resistance and high recurrence after treatment [1, 2]. Emerging evidence has suggested that aberrant epigenetic changes, such as DNA methylation, may represent an important mechanism governing tumor progression and drug resistance $[10,46]$. Due to its dynamic nature in response to physiological and microenvironmental stimuli[47], DNA methylation has served as a potential biomarker associated with cancer diagnosis, drug resistance, and tumor progression $[48,49]$. Therefore, we performed whole epigenome profiling analysis for the global methylation status of a retrospective cohort of 80 primary tumor samples of EOC. Our analysis demonstrated that global DNA hypermethylation is preferentially found in high-grade EOC, indicating that the activities of PI3KAKT, MAPK, RAS, and WNT oncogenic pathways could be altered that may be in favor of tumor aggressiveness, shorter overall survival (OS), and a high mortality rate. In 30 HGSOC samples, a high level of global DNA methylation was observed specifically in the recurrent cases, indicating that DNA methylation is associated with acquired platinum resistance. Consistent with this result, targeting DNA methylation by using the clinically approved DNA demethylating agents such as azacytidine, decitabine, and thioguanine could notably attenuate the aggressiveness of EOC and synergistically enhance cisplatin-mediated cell cytotoxicity in HGSOC cells. Our results underscore the important role played by global DNA methylation in tumor progression and platinum resistance in EOC.

It is well known that DNA methyltransferases (DNMTs) and histone deacetylases (HDACs) are crucially involved in DNA methylation, which promotes tumor development and progression [50]. Recent evidence has shown that three DNMTs, DNMT1, DNMT3A, and DNMT3B, are frequently upregulated in aggressive EOC [51]. In keeping with these reports, our EWAS analysis demonstrated that a higher level of global DNA methylation was significantly associated with high-grade EOC, confirming that higher gene silencing events are involved in the aggressiveness of EOC. This association was supported by 9313 DMPs mapped to their relative gene regions (promoter, gene body, 3'UTR) of 4938 genes potentially silenced by DNA methylation. On the other hand, our analysis also showed hypomethylated DMPs for some genes, confirming previous reports showing that upregulated UHRF1/2 could mediate DNMT3A ubiquitination and proteasome-dependent degradation, leading to genome-wide de novo DNA methylation in cancer cells [52, 53]. However, hypermethylated DMPs were still dominant in high-grade EOC, leading to gene silencing for not only TSGs or inhibitors of small GTPases, such as TIMP1, RHOH, EphB2, and ARRB1 [25-27], but also 


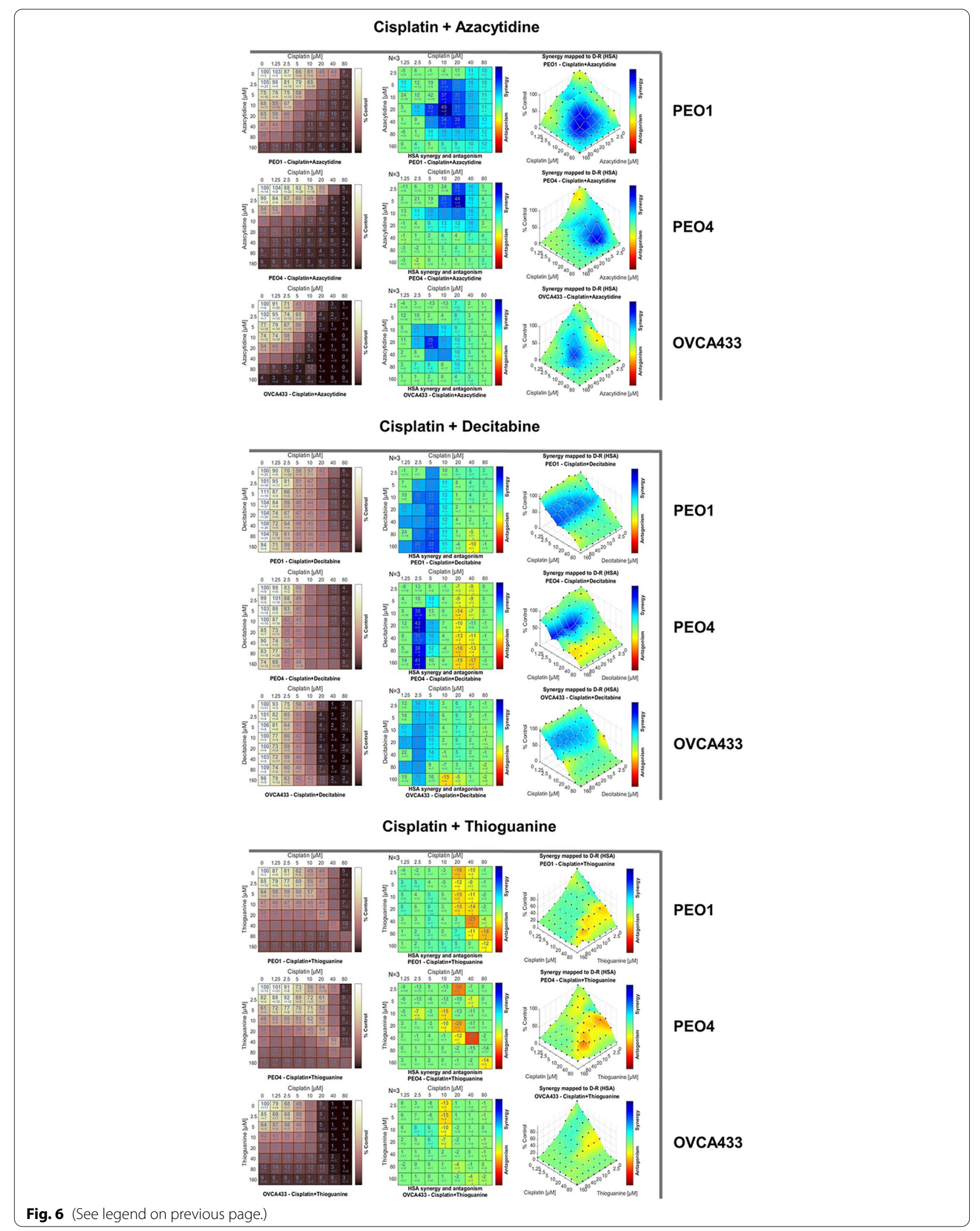


key TSGs of numerous oncogenic signaling pathways, e.g., PTEN and PIK3R1 of the PI3K-AKT signaling pathway [28, 29], EFNA3 and NF1 of the MAPK signaling pathway [30,31], $A B L 1$ and $P A K 1$ of the RAS signaling pathway [32], PRICKLE1 and DAAM1 [33]. Consistently, these aberrantly activated oncogenic pathways have been reported to promote tumor aggressiveness, drug resistance, and poor OS in high-grade EOC [54].

Our study identified 7 DMP-associated genes that could be a DNA methylation signature for determining high-grade and aggressive EOC. Of these 7 DMP-associated genes, hypomethylated CLDN16 is a member of the claudin family modulating cell polarity and is also a biomarker of breast cancer aggressiveness [55]. Additionally, hypermethylated LZTFL1 has been reported to be involved in maintaining cell differentiation [56], ABHD2 was found to control anoikis resistance in HGSOC [57], RNF144A was shown to be a tumor suppressor in governing breast cancer growth and metastasis [58], and HPS4 was shown to alter the functions of the small GTPases Rab32 and Rab38, which are associated with the recurrence of lung cancer [59], 60. Although another 2 genes have not been reported to have functions in cancers, these DMP-associated genes have been inferred to be putative DNA methylation signatures for predicting the high grade and aggressiveness of EOC. On the other hand, we also identified 8 DMP-associated genes that could be used to determine tumor recurrence and shorter overall survival (OS). Of these 8 DMP-associated genes, CLDN16 and PIK3C2G contained DNA-hypomethylated DMPs, while WWP1, DISP1, FNDC3B, NRIP1, FDE4D, and CLDN12 were enriched by DNA-hypermethylated DMPs. In the comparison of the hub gene lists, only CLDN16 was on both lists. However, these 8 DMP-associated genes were found only from 200 DMPs encoding 177 genes, indicating that these 8 DMP-associated genes are less well-supported for use as a DNA methylation signature to predict tumor recurrence and overall survival (OS) of EOC. Further investigation using large-scale combined analyses of large-scale retrospective and prospective occurrences in EOC cohort studies is warranted.

There is an ever-growing body of evidence indicating that aberrant epigenetic alterations critically govern tumor heterogeneity, tumorigenesis, and drug resistance [61, 62]. For example, DNA methylation-mediated gene silencing of checkpoint kinase 2 (CHK2) was found to be associated with cisplatin resistance in non-small-cell lung carcinoma (NSCLC) [63]. Similarly, DNA hypermethylation of the mismatch repair gene hMSH2 was also reported in platinum-resistant EOC [64]. In fact, global DNA methylation changes leading to drug resistance have been frequently observed in various solid tumors [35]. Clinically, the responses to platinum-based chemotherapy in HGSOC can be classified into platinum-refractory, platinum-resistant and platinum-sensitive groups [65]. In this study, we excluded patients with platinum-refractory outcomes. The reasons were that this group has a complete lack of response to the first line of platinum-/taxane-based therapy, and the mechanisms for this lack of effect may include the mixed events of cancer stem cells (CSCs), genetic mutations, and epigenetic alterations $[66,67]$. Indeed, this group clinically shows a significant difference from other serous subtype tumors in responding to platinum-based therapy and progression during platinum-based therapy [66]. Consistent with this finding, our EWAS found that the platinum-refractory group was different from other platinum-resistant and platinum-sensitive groups (data not shown). In contrast, our findings demonstrated that HGSOC with higher global DNA methylation usually has recurrence, implying that DNA methylation is associated with platinum resistance. In contrast to other well-known oncogenic signaling pathways in platinum resistance, such as the DNA repair pathway[68], our analysis found that the most common hypermethylation-associated genes, such as OR51L1, OR51I1, OR51F1, OR51B6, HBBP1, TMEM200A, and DLG2, are associated with olfactory transduction, neuroactive ligand-receptor interaction, calcium signaling, and cAMP signaling pathways. These pathways are very uncommon oncogenic pathways and are rarely reported in cancer drug resistance. However, emerging evidence has shown that DNA hypermethylation could affect the activities of olfactory receptor pathways regulating cancer cell proliferation and apoptosis [69], while calcium signaling and cAMP signaling pathways in association with neurotransmitters or inflammation-associated molecules could enhance ovarian cancer metastasis and drug resistance [70]. These findings suggest that further research be conducted to confirm that these DNA methylation pathways are associated with acquired drug resistance, and this study may guide new avenues of targeted therapy for recurrent HGSOC.

Targeting DNA methylation for epigenetic therapy has received increasing attention from cancer researchers [71]. Although two DNA demethylating agents, 5-azacytidine and 5-aza-2'-deoxycytidine (decitabine), have been approved for clinical use to date, hundreds of clinical trials from commercial DNA demethylating agents have examined the anticancer effect on various cancer types. However, there are accumulating examples showing variable anti-DNA methylation functions in different cancer cell types [72]. In this study, we tested three commercially available demethylating agents, azacytidine [73], decitabine [39], and thioguanine [74], on the proliferation and platinum resistance of EOC cells. As expected, these three demethylating agents could inhibit the proliferation 
of EOC cells by different mechanisms; azacytidine primarily enhanced cell apoptosis, while decitabine and thioguanine preferentially induced G2/M cell cycle arrest. However, only azacytidine and decitabine synergistically enhanced cisplatin-mediated cytotoxicity. This finding suggests that the selection of suitable DNA demethylating agents is very important for optimizing anticancer effects and minimizing platinum resistance.

This study is a retrospective study with a relative small sample size. The DNA methylation biomarkers identified in EWAS are still needed to have prospective validation to increase their clinical predictive value. In addition, although we have demonstrated DNA demethylating agents possess remarkable effects on anticancer and antidrug resistance, histone modifications also closely interact with DNA demethylation in silencing gene expression in cancer progression. In future research, we should test the effect of HDAC inhibitors, such as SAHA (vorinostat) and romidepsin, on the reversibility of gene silencing and consider dual-targeted therapy.

\section{Conclusions}

Our EWAS showed that a high level of global DNA methylation is associated with the aggressiveness of high-grade EOC and the acquired platinum resistance of HGSOC. Careful selection of demethylating agents for anti-DNA methylation is required to obtain better anticancer treatment and reduced platinum resistance in HGSOC.

\begin{abstract}
Abbreviations
ATCC: American Type Culture Collection; CPOS: Centre for PanorOmic Sciences; DMPs: Differentially methylated probes; DMRs: Differentially methylated regions; DMEM: Dulbecco's modified Eagle's medium; EOC: Epithelial ovarian cancer; EWAS: Epigenome-wide association study; FBS: Fetal bovine serum; GO: Gene Ontology; hbFGF: Human basic fibroblast growth factor; hEGF: Human epidermic growth factor; HGSOC: High-grade serous ovarian carcinoma; HG: High-grade; KEGG: Kyoto Encyclopedia of Genes and Genomes; LG: Low-grade; OCT: Optimal cutting temperature; OvCa: Ovarian cancer; OS: Overall survival; PI: Propidium iodide; RPMI: Roswell Park Memorial Institute; TSGs: Tumor suppressor genes.
\end{abstract}

\section{Supplementary Information}

The online version contains supplementary material available at https://doi. org/10.1186/s13148-021-01130-5.

Additional file 1: Table S1. The top 17 DMP-associated genes (screened by the enrichment of DMPs on their promoter regions) on the 22 autosomal chromosomes according to the analysis of 14,120 DMPs at a false discovery rate (FDR) threshold $\left(\mathrm{P}<5^{*} 10^{-11}\right)$.

Additional file 2: Table S2. The list of differentially methylated genes that are involved in modulating small GTPase activities according to the 9313 DMPs in GO enrichment analysis.

Additional file 3: Table S3. According to the KEGG pathway, the list of differentially methylated genes is determined by 9313 DMPs and is involved in the PI3K-AKT, MAPK, RAS, and WNT cancer signaling pathways analysis.
Additional file 4: Table S4. The top 20 DMP-associated genes (screened by the enrichment of DMPs on their promoter regions) on the 22 autosomal chromosomes according to the analysis of 2969 DMPs at a false discovery rate (FDR) threshold $\left(P<5^{*} 10^{-3}\right)$.

Additional file 5: Fig. S1. Kaplan-Meier estimate of OS using methylation signature genes (CLDN16, LZTFL1, FARS2, SGMS2, ABHD2, RNF144A, and HPS4).

Additional file 6: Fig. S2. Boxplot visualization of comparison of the DNA methylation level of tumor grading. EOC samples were marked by tumor stage, tumor subtype, recurrence, and survival status.

Additional file 7: Fig. S3. Heatmap visualization of DNA methylation levels in the PI3K-AKT, RAS, MAPK, and WNT signaling pathways and KaplanMeier estimates of OS using the methylation signature of genes.

Additional file 8: Fig. S4. Gene ontology (GO) enrichment analysis of the overall functional relevance of genes associated with DMPs using the clusterProfiler package in $\mathrm{R}$.

Additional file 9: Fig. S5. (A) Table summarizing the IC50 values of a 48-h treatment with cisplatin, azacytidine, decitabine, and thioguanine in the six ovarian cancer cell lines using the XTT assay. IC50 values were determined at the drug concentration leading to a $50 \%$ viability reduction compared to the control. (B) Annexin V-PI staining of A2780cp and A2780s cells after a 48-h treatment with different concentrations of cisplatin, azacytidine, decitabine, and thioguanine by flow cytometry. Data analysis was performed with CytExpert software. (C) Population of apoptotic cells in each treatment condition from Annexin V-PI staining. The apoptotic cell population was represented by the cell population in the lower right quadrant (early apoptotic) and upper right quadrant (late apoptotic). (D) Cell cycle analysis of A2780cp and A2780s cells after $48 \mathrm{~h}$ of treatment with different concentrations of cisplatin, azacytidine, decitabine, and thioguanine by flow cytometry. Data analysis was performed with Modfit LT software. (E) Distribution of cells in different phases of the cell cycle in each treatment condition. Populations of cells distributed in G0/G1, S, G2/M and SubG1 phases are presented in different colored peaks.

\section{Acknowledgements}

We thank The University of Hong Kong, LKS Faculty of Medicine, Centre for PanorOmic Sciences (CPOS), Genomics Core for sample processing and beadchip scanning.

\section{Authors' contributions}

DC, MY, YC, WC, and FH carried out genomic DNA isolation, 3D cell culture, cell experiments, and drug testing assays. FC participated all bioinformatic analysis. DC, WL, BL, and HN conceived of the study and participated in its design. DC, WL, SL, KC, and HN contributed and analyzed clinical samples and clinicopathological data. DC, WL, FC, BL, and $\mathrm{HN}$ analyzed and interpreted data in this study. DC, FC, YC, and MY helped to draft the manuscript. All authors read and approved the final manuscript.

\section{Funding}

This study was supported by University-Industry Collaboration Programme, the Innovation and Technology Commission, (Hong Kong) (UIM367).

\section{Availability of data and materials}

All methylome profiling data associated with this paper are deposited into GEO repository with the accession number GSE168930 (https://www.ncbi.nlm. nih.gov/geo/query/acc.cgi?acc=GSE168930). The other data supporting the findings of the present study are available from the corresponding author on reasonable request.

\section{Declarations}

\section{Ethical approval and consent to participate}

This study was approved by the Institutional Review Board of the University of Hong Kong/Hospital Authority Hong Kong West Cluster (HKU/HA HKW IRS) (IRS Reference Number: UW 11-298), and the written informed consent was 
obtained from each patient. We confirmed all the data used in this study was anonymized or maintained with confidentiality.

\section{Consent for publication}

All authors have approved the manuscript for publication.

\section{Competing interests}

Drs. Benjamin Li and Wai-Yip Lam are from Lee's Pharmaceutical (HK) Ltd. The other authors declare no conflict of interest.

\section{Author details}

'Department of Obstetrics and Gynaecology, L747 Laboratory Block, LKS Faculty of Medicine, 21 Sassoon Road, Pokfulam, Hong Kong, SAR, People's Republic of China. 'Lee's Pharmaceutical (HK) Ltd, 1/F Building 20E, Phase 3, Hong Kong Science Park, Shatin, Hong Kong, People's Republic of China. ${ }^{3}$ Department of Obstetrics and Gynaecology, 6/F Professorial Block, Queen Mary Hospital, Pokfulam, Hong Kong, People's Republic of China.

Received: 13 April 2021 Accepted: 12 July 2021

Published online: 22 July 2021

\section{References}

1. Lheureux S, Braunstein M, Oza AM. Epithelial ovarian cancer: Evolution of management in the era of precision medicine. CA Cancer J Clin. 2019;69(4):280-304

2. Chandra A, Pius C, Nabeel M, et al. Ovarian cancer: Current status and strategies for improving therapeutic outcomes. Cancer Med. 2019;8(16):7018-31

3. Narod SA, Boyd J. Current understanding of the epidemiology and clinical implications of BRCA1 and BRCA2 mutations for ovarian cancer. Curr Opin Obstet Gynecol. 2002;14(1):19-26.

4. Kobel M, Reuss A, du Bois A, et al. The biological and clinical value of p53 expression in pelvic high-grade serous carcinomas. J Pathol. 2010;222(2):191-8.

5. Della Pepa C, Tonini G, Santini D, et al. Low Grade Serous Ovarian Carcinoma: from the molecular characterization to the best therapeutic strategy. Cancer Treat Rev. 2015;41(2):136-43.

6. Chornokur G, Lin HY, Tyrer JP, et al. Common genetic variation in cellular transport genes and epithelial ovarian cancer (EOC) risk. PLOS ONE. 2015;10(6):e0128106.

7. Zorn KK, Bonome T, Gangi L, et al. Gene expression profiles of serous, endometrioid, and clear cell subtypes of ovarian and endometrial cancer. Clin Cancer Res. 2005;11(18):6422-30.

8. Li C, Bonazzoli E, Bellone S, et al. Mutational landscape of primary, metastatic, and recurrent ovarian cancer reveals c-MYC gains as potential target for BET inhibitors. Proc Natl Acad Sci U S A. 2019;116(2):619-24.

9. Kim YM, Lee SW, Chun SM, et al. Analysis and comparison of somatic mutations in paired primary and recurrent epithelial ovarian cancer samples. PLoS ONE. 2014;9(6):e99451.

10. Korbel J, Roberts C. (2017) A convergence of genetics and epigenetics in cancer. Cell. 2017;168(4):561-3.

11. Chatterjee A, Rodger EJ, Eccles MR. Epigenetic drivers of tumourigenesis and cancer metastasis. Semin Cancer Biol. 2018;51:149-59.

12. Lengyel E. Ovarian cancer development and metastasis. Am J Pathol. 2010;177(3):1053-64.

13. Razin A, Riggs AD. DNA methylation and gene function. Science. 1980;210(4470):604-10

14. Momparler RL, Bovenzi V. DNA methylation and cancer. J Cell Physiol. 2000;183(2):145-54.

15. Jackson K, Yu MC, Arakawa K, et al. DNA hypomethylation is prevalent even in low-grade breast cancers. Cancer Biol Ther. 2004;3(12):1225-31.

16. Delcuve GP, Rastegar M, Davie JR. Epigenetic control. J Cell Physiol. 2009;219(2):243-50.

17. Fardi M, Solali S, Farshdousti HM. Epigenetic mechanisms as a new approach in cancer treatment: an updated review. Genes Dis. 2018;5(4):304-11.

18. Ehrlich M. DNA hypermethylation in disease: mechanisms and clinical relevance. Epigenetics. 2019;14(12):1141-63.
19. Tian Z, Meng L, Long X, et al. DNA methylation-based classification and identification of bladder cancer prognosis-associated subgroups. Cancer Cell Int. 2020;20:255.

20. Chen W, Zhuang J, Wang PP, et al. DNA methylation-based classification and identification of renal cell carcinoma prognosis-subgroups. Cancer Cell Int. 2019;19:185.

21. Hakulinen T, Abeywickrama KH. A computer program package for relative survival analysis. Comput Programs Biomed. 1985;19(2-3):197-207.

22. Therneau TM, Li H. Computing the Cox model for case cohort designs. Lifetime Data Anal. 1999;5(2):99-112.

23. Zhang MJ. Cox proportional hazards regression models for survival data in cancer research. Cancer Treat Res. 2002;113:59-70.

24. Jones PA. Functions of DNA methylation: islands, start sites, gene bodies and beyond. Nat Rev Genet. 2012;13(7):484-92.

25. Kandouz M, Haidara K, Zhao J, et al. The EphB2 tumor suppressor induces autophagic cell death via concomitant activation of the ERK1/2 and PI3K pathways. Cell Cycle. 2010;9(2):398-407.

26. Wang YS, Tzeng HT, Tsai $\mathrm{CH}$, et al. VAMP8, a vesicle-SNARE required for RAB37-mediated exocytosis, possesses a tumor metastasis suppressor function. Cancer Lett. 2018:437:79-88.

27. Li X, Bu X, Lu B, et al. The hematopoiesis-specific GTP-binding protein RhoH is GTPase deficient and modulates activities of other Rho GTPases by an inhibitory function. Mol Cell Biol. 2002;22(4):1158-71.

28. Vallejo-Diaz J, Chagoyen M, Olazabal-Moran M, et al. The Opposing roles of PIK3R1/p85alpha and PIK3R2/p85beta in cancer. Trends Cancer. 2019;5(4):233-44.

29. Goberdhan DC, Wilson C. PTEN: tumour suppressor, multifunctional growth regulator and more. Hum Mol Genet. 2003;12 Spec No 2:R239-48.

30. Lau N, Feldkamp MM, Roncari L, et al. Loss of neurofibromin is associated with activation of RAS/MAPK and PI3-KAKT signaling in a neurofibromatosis 1 astrocytoma. J Neuropathol Exp Neurol. 2000;59(9):759-67.

31. Wang Z, Liu Z, Liu B, et al. Dissecting the roles of Ephrin-A3 in malignant peripheral nerve sheath tumor by TALENs. Oncol Rep. 2015;34(1):391-8.

32. Dasgupta Y, Koptyra M, Hoser G, et al. Normal ABL1 is a tumor suppressor and therapeutic target in human and mouse leukemias expressing oncogenic ABL1 kinases. Blood. 2016;127(17):2131-43.

33. Chan DW, Chan CY, Yam JW, et al. Prickle-1 negatively regulates Wnt/ beta-catenin pathway by promoting Dishevelled ubiquitination/degradation in liver cancer. Gastroenterology. 2006;131(4):1218-27.

34. Fortner RT, Poole EM, Wentzensen NA, et al. Ovarian cancer risk factors by tumor aggressiveness: an analysis from the Ovarian Cancer Cohort Consortium. Int J Cancer. 2019:145(1):58-69.

35. Romero-Garcia S, Prado-Garcia H, Carlos-Reyes A. Role of DNA Methylation in the Resistance to Therapy in Solid Tumors. Front Oncol. 2020;10:1152.

36. Slaughter K, Holman LL, Thomas EL, et al. Primary and acquired platinumresistance among women with high grade serous ovarian cancer. Gynecol Oncol. 2016;142(2):225-30.

37. Kim S, Han Y, Kim SI, et al. Tumor evolution and chemoresistance in ovarian cancer. NPJ Precis Oncol. 2018;2:20.

38. Seelan RS, Mukhopadhyay P, Pisano MM, et al. Effects of 5-Aza-2'deoxycytidine (decitabine) on gene expression. Drug Metab Rev. 2018;50(2):193-207.

39. Kantarjian H, Issa JP, Rosenfeld CS, et al. Decitabine improves patient outcomes in myelodysplastic syndromes: results of a phase III randomized study. Cancer. 2006;106(8):1794-803.

40. Yuan B, Zhang J, Wang $\mathrm{H}$, et al. 6-Thioguanine reactivates epigenetically silenced genes in acute lymphoblastic leukemia cells by facilitating proteasome-mediated degradation of DNMT1. Cancer Res. 2011;71(5):1904-11.

41. Williams CJ, Whitehouse JM. Cis-platinum: a new anticancer agent. Br Med J. 1979;1(6179):1689-91.

42. Domcke S, Sinha R, Levine DA, et al. Evaluating cell lines as tumour models by comparison of genomic profiles. Nat Commun. 2013:4:2126.

43. Yang $X$, Fraser M, Moll UM, et al. Akt-mediated cisplatin resistance in ovarian cancer: modulation of p53 action on caspase-dependent mitochondrial death pathway. Cancer Res. 2006;66(6):3126-36.

44. Goyeneche A, Lisio MA, Fu L, et al. The capacity of high-grade serous ovarian cancer cells to form multicellular structures spontaneously along disease progression correlates with their orthotopic tumorigenicity in immunosuppressed mice. Cancers (Basel). 2020;12(3):699. 
45. Coscia F, Watters KM, Curtis M, et al. Integrative proteomic profiling of ovarian cancer cell lines reveals precursor cell associated proteins and functional status. Nat Commun. 2016;7:12645.

46. Moore LD, Le T, Fan G. DNA methylation and its basic function. Neuropsychopharmacology. 2013;38(1):23-38.

47. Dowen RH, Pelizzola M, Schmitz RJ, et al. Widespread dynamic DNA methylation in response to biotic stress. Proc Natl Acad Sci U S A. 2012;109(32):E2183-91.

48. Maeda O, Ando T, Ohmiya N, et al. Alteration of gene expression and DNA methylation in drug-resistant gastric cancer. Oncol Rep. 2014;31(4):1883-90.

49. Locke WJ, Guanzon D, Ma C, et al. DNA Methylation Cancer Biomarkers: Translation to the Clinic. Front Genet. 2019;10:1150.

50. Robertson KD. DNA methylation, methyltransferases, and cancer. Oncogene. 2001;20(24):3139-55.

51. GuY, Yang P, Shao Q, et al. Investigation of the expression patterns and correlation of DNA methyltransferases and class I histone deacetylases in ovarian cancer tissues. Oncol Lett. 2013;5(2):452-8.

52. Jia $Y, L i P$, Fang $L$, et al. Negative regulation of DNMT3A de novo DNA methylation by frequently overexpressed UHRF family proteins as a mechanism for widespread DNA hypomethylation in cancer. Cell Discov. 2016;2:16007.

53. Hong JH, Jin EH, Kim S, et al. LINE-1 hypomethylation is inversely correlated with UHRF1 overexpression in gastric cancer. Oncol Lett. 2018;15(5):6666-70.

54. Kotrbova A, Ovesna P, Gybel T, et al. WNT signaling inducing activity in ascites predicts poor outcome in ovarian cancer. Theranostics. 2020;10(2):537-52.

55. Kuo SJ, Chien SY, Lin C, et al. Significant elevation of CLDN16 and HAPLN3 gene expression in human breast cancer. Oncol Rep. 2010;24(3):759-66.

56. Wei Q, Chen ZH, Wang L, et al. LZTFL1 suppresses lung tumorigenesis by maintaining differentiation of lung epithelial cells. Oncogene. 2016;35(20):2655-63.

57. Yamanoi K, Matsumura N, Murphy SK, et al. Suppression of ABHD2, identified through a functional genomics screen, causes anoikis resistance, chemoresistance and poor prognosis in ovarian cancer. Oncotarget. 2016;7(30):47620-36.

58. Yang YL, Zhang Y, Li DD, et al. RNF144A functions as a tumor suppressor in breast cancer through ubiquitin ligase activity-dependent regulation of stability and oncogenic functions of HSPA2. Cell Death Differ 2020;27(3):1105-18

59. Gerondopoulos A, Langemeyer L, Liang JR, et al. BLOC-3 mutated in Hermansky-Pudlak syndrome is a Rab32/38 guanine nucleotide exchange factor. Curr Biol. 2012;22(22):2135-9.

60. Hsieh JJ, Hou MM, Chang JW, et al. RAB38 is a potential prognostic factor for tumor recurrence in non-small cell lung cancer. Oncol Lett. 2019;18(3):2598-604.
61. Chen QW, Zhu XY, Li YY, et al. Epigenetic regulation and cancer (review). Oncol Rep. 2014;31(2):523-32.

62. Easwaran H, Tsai HC, Baylin SB. Cancer epigenetics: tumor heterogeneity, plasticity of stem-like states, and drug resistance. Mol Cell. 2014;54(5):716-27.

63. Zhang P, Wang J, Gao W, et al. CHK2 kinase expression is down-regulated due to promoter methylation in non-small cell lung cancer. Mol Cancer. 2004;3:14.

64. Tian $H$, Yan $L$, Xiao-Fei L, et al. Hypermethylation of mismatch repair gene hMSH2 associates with platinum-resistant disease in epithelial ovarian cancer. Clin Epigenetics. 2019;11(1):153.

65. Matsuo K, Lin YG, Roman LD, et al. Overcoming platinum resistance in ovarian carcinoma. Expert Opin Investig Drugs. 2010;19(11):1339-54.

66. Pujade-Lauraine E, Banerjee S, Pignata S. Management of Platinumresistant, relapsed epithelial ovarian cancer and new drug perspectives. J Clin Oncol. 2019;37(27):2437-48.

67. Cooke SL, Brenton JD. Evolution of platinum resistance in high-grade serous ovarian cancer. Lancet Oncol. 2011;12(12):1169-74.

68. Damia G, Broggini M. Platinum resistance in ovarian cancer: role of DNA repair. Cancers (Basel). 2019:11(1):119.

69. Giri AK, Aittokallio T. DNMT inhibitors increase methylation in the cancer genome. Front Pharmacol. 2019;10:385.

70. Predescu DV, Cretoiu SM, Cretoiu D, et al. G Protein-coupled receptors (GPCRs)-mediated calcium signaling in ovarian cancer: Focus on GPCRs activated by neurotransmitters and inflammation-associated molecules. Int J Mol Sci. 2019;20(22):5568.

71. Wang J, Yang J, Li D, et al. Technologies for targeting DNA methylation modifications: Basic mechanism and potential application in cancer. Biochim Biophys Acta Rev Cancer. 2021;1875(1):188454.

72. Cheng $Y$, He C, Wang M, et al. Targeting epigenetic regulators for cancer therapy: mechanisms and advances in clinical trials. Signal Transduct Target Ther. 2019;4:62.

73. Antonsson BE, Avramis VI, Nyce J, et al. Effect of 5-azacytidine and congeners on DNA methylation and expression of deoxycytidine kinase in the human lymphoid cell lines CCRF/CEM/0 and CCRF/CEM/dCk-1. Cancer Res. 1987:47(14):3672-8.

74. Nelson JA, Carpenter JW, Rose LM, et al. Mechanisms of action of 6-thioguanine, 6-mercaptopurine, and 8-azaguanine. Cancer Res. 1975;35(10):2872-8.

\section{Publisher's Note}

Springer Nature remains neutral with regard to jurisdictional claims in published maps and institutional affiliations.
Ready to submit your research? Choose BMC and benefit from:

- fast, convenient online submission

- thorough peer review by experienced researchers in your field

- rapid publication on acceptance

- support for research data, including large and complex data types

- gold Open Access which fosters wider collaboration and increased citations

- maximum visibility for your research: over $100 \mathrm{M}$ website views per year

At $\mathrm{BMC}$, research is always in progress.

Learn more biomedcentral.com/submissions 